\title{
Efectos subletales y costos de resistencia a abamectina en palomilla dorso de diamante (Plutella xylostella) (Lepidoptera: Plutellidae)
}

\author{
Sublethal effects and costs of resistance to abamectin in diamondback moth \\ (Plutella xylostella) (Lepidoptera: Plutellidae)
}

\author{
(iD) JOSÉ FRANCISCO RODRÍGUEZ-RODRÍGUEZ ${ }^{1}$; (D) ERNESTO \\ CERNA-CHÁVEZ ${ }^{*}$; iD YISA MARÍA OCHOA-FUENTES ${ }^{1}$; iD JERÓNIMO \\ LANDEROS-FLORES ${ }^{1}$; ID LUIS PATRICIO GUEVARA-ACEVEDO²; \\ (iD HUGO CESAR CISNEROS-LÓPEZ ${ }^{2}$
}

\footnotetext{
${ }^{1}$ Universidad Autónoma Agraria Antonio Narro, Coahuila, México,francisco_azul@live.com.mx,jabaly1@yahoo.com,yisa8a@yahoo.com,jlanflo@hotmail. com²Instituto Nacional de México, Instituto Tecnológico de Roque, Celaya, Guanajuato, México, lpguevara@itroque.edu.mx, hugo.cl@roque.tecnm.mx
}

\begin{abstract}
Autor para correspondencia
Ernesto Cerna-Chávez. Doctor en ciencias, Universidad Autónoma Agraria Antonia Narro, Calzada Antonio Narro 1923, Buenavista, Saltillo, C.P. 25315, Coahuila México, jabaly1@ yahoo.com

\section{Citación sugerida}

RODRÍGUEZ-RODRÍGUEZ, J. F.; CERNACHÁVEZ, E.; OCHOA-FUENTES, Y. M.; LANDEROS-FLORES, J.; GUEVARA-ACEVEDO, L. P.; CISNEROS-LÓPEZ, H. C. 2021. Efectos subletales y costos de resistencia a abamectina en palomilla dorso de diamante (Plutella xylostella) (Lepidoptera: Plutellidae). Revista Colombiana de Entomología 47 (2): e10657. https://doi.org/10.25100/socolen. v47i2.10657
\end{abstract}

Recibido: 02-oct-2020

Aceptado: 12-feb-2021

Publicado: 19-ago-2021

Revista Colombiana de Entomología ISSN (Print): 0120-0488

ISSN (On Line): 2665-4385

https://revistacolombianaentomologia.univalle.edu.co

Open access

(c) (1) (2) (2) BY-NC-SA 4.0

Publishers: Sociedad Colombiana de Entomología SOCOLEN (Bogotá, D. C., Colombia) https://www.socolen.org.co

Universidad del Valle (Cali, Colombia)

https://www.univalle.edu.co

(C) 2021 Sociedad Colombiana de Entomología SOCOLEN y Universidad del Valle - Univalle
Resumen: Es fundamental comprender los efectos que el uso de concentraciones subletales de insecticidas puede tener en la dinámica, biología y el desarrollo de plagas como Plutella xylostella, una de las plagas de mayor importancia a nivel mundial. Esta especie tiene la capacidad de desarrollar resistencia fácilmente a los insecticidas utilizados para su control. En el presente estudio, se realizó una presión de selección de abamectina durante diez generaciones de P. xylostella y se determinaron las concentraciones subletales $\left(\mathrm{CL}_{20}\right)$ de las líneas resistente y susceptible. Luego, estas concentraciones se aplicaron a larvas de tercer instar y se evaluó el efecto sobre el porcentaje de pupas formadas, peso de pupas, emergencia de adultos, en dos generaciones, así como los tiempos de desarrollo de cada una de las fases desde huevo hasta adulto. La resistencia y la aplicación de concentraciones subletales presenta costos importantes en la biología y desarrollo de $P$. xylostella al aumentar el porcentaje de pupación de una generación a otra, así como un mayor peso de pupas, un mayor número de huevos por hembra/día. Sin embargo, se presenta un efecto transgeneracional al disminuir la oviposición en sus descendientes; en lo que se refiere al tiempo de desarrollo, presentó un efecto nocivo al prolongarlo en más de siete días lo que generaría un mayor número de aplicaciones por ciclo.

Palabras clave: Efectos subletales, control de plagas, abamectina, resistencia, Plutella xylostella.

Abstract: It is essential to understand the effects of sublethal concentrations of insecticides have on the dynamics, biology, and development of pests such as Plutella xylostella, one of the most important pests in the world. This species can develop resistance to the insecticides used for its control. In the present study, abamectin selection pressure was performed for ten generations of P. xylostella, and the sublethal concentrations $\left(\mathrm{CL}_{20}\right)$ and susceptible lines of the resistance were determined. Then, these concentrations were applied to third instar larvae. In two generations it was evaluated the effect on the percentage of pupae formed, pupal weight, adult emergence, and the development times of each phase from egg to adult. The resistance and application of sublethal concentrations have considerable costs in the biology and development of $P$. xylostella by increasing the percentage of pupation from one generation to another, and a greater weight of pupae and eggs per female/day. However, it presents a transgenerational effect by reducing oviposition in its generation; in terms of development time, it presented a harmful effect by prolonging it by more than seven days, which would generate a greater number of applications per cycle.

Keywords: Sublethal effects, pest control, abamectin, resistance, Plutella xylostella.

\section{Introducción}

La palomilla del dorso del diamante (PDD), Plutella xylostella (Linnaeus, 1758) (Lepidoptera: Plutellidae), es una de las plagas de insectos más destructivas de los cultivos brassicaceas en todo el mundo (Furlong et al. 2013). En su fase larval se alimenta las partes frondosas de la planta y llega provocar pérdidas de rendimiento de aproximadamente 50-80 \% durante la infestación (Grzywacz et al. 2010), que se refleja en pérdidas económicas de U\$ 4-5 mil millones anuales (Furlong et al. 2013). 
Esta especie es una plaga particularmente dañina debido a su alta tasa de reproducción y su capacidad para desarrollar resistencia a los insecticidas utilizados para su control (Wang y Wu 2012), entre ellos: cipermetrina, abametina, indoxacar, spinosad, chlorantranilipole y Bacillus thuringiensis (Arthropod Pesticide Resistance Database 2020). La abemectina es uno de los insecticidas más usados para el control de $P$. xylostella (Wang y Wu 2014). Este producto es derivado de fermentación natural de la bacteria del suelo Streptomyces avermitilis (Memarizadeh et al. 2013). Actúa sobre la transmisión nerviosa del insecto: se fija sobre un receptor glutamato de los canales de cloro de la membrana de las células nerviosas, cerca del receptor GABA y de un receptor de benzodiacepinas, minimizando la acción del GABA, lo que genera un aumento en la liberación de este, dando lugar a un potencial de acción (García Salazar et al. 2011); su fijación provoca un flujo de iones $\mathrm{Cl}^{-}$al interior de las células nerviosas del insecto, origina su hiperpolarización y una falta de respuesta a los estímulos clásicos, provocando la muerte (Bowman, 2021). Investigaciones previas reportan efectos subletales asociados a la resistencia y aplicación de dosis subletales de abamaectina en un mayor tiempo de desarrollo de pupas, menor peso de pupas y menor supervivencia larval, así como la fecundidad de las hembras y la viabilidad del huevo se disminuyen (Wang y Wu 2014). Xu et al. (2010) mencionan que hembras resistentes a la abamectina producen menos feromona sexual y muestran un nivel más bajo de comportamiento de llamada, mientras que los machos resistentes presentaron una mayor capacidad de respuesta a las feromonas sexuales de hembras. Efectos similares se han reportado en otras plagas. El caso de Tuta absoluta Meyrick, 1917 (Lepidoptera: Gelichiidae) al ser expuesta a dosis subletales de este insecticida, se disminuyó la emergencia del adulto, el porcentaje de pupación, la fecundidad y la longevidad del adulto (Zibaee y Esmaeily 2017). Por su parte Saber et al. (2018) mencionan que la aplicación de dosis subletales de abamectina sobre el ácaro de dos manchas (Tetranychus urticae C. L. Koch, 1836 Prostigmata: Tetranychidae) afecta su tiempo de desarrollo, la tasa de supervivencia y la fecundidad.

Los insecticidas pueden bloquear algunos procesos fisiológicos o bioquímicos al afectar la supervivencia, el crecimiento, el desarrollo, la reprodución y el comportamiento de los insectos (De Castro et al. 2013). El uso de dosis/concentraciones subletales de agentes tóxicos llegan afectar la reproducción, orientación, alimentación, oviposición y aprendizaje de las plagas (Saran et al. 2014). Además, Guedes y Cutler (2013) mencionan que, existe la posibilidad de que ocurra hormesis, fenómeno conocido en poblaciones expuestas a dosis subletales. A esta respuesta por parte de las poblaciones se le considera como una estimulación del rendimiento de los organismos que se produce a bajos niveles de exposición a agentes químicos que normalmente son tóxicos en niveles altos (Calabrese y Baldwin 2001), y es considerado como el principal mecanismo para el resurgimiento de plagas (Cordeiro et al. 2013; Qu et al. 2015). Es fundamental comprender cómo la exposición a compuestos químicos afecta la dinámica de la población de especies de plagas económicamente importantes, para desarrollar esquemas efectivos de manejo integrado de plagas (Banks et al. 2017). El objetivo de la presente investigación fue evaluar el efecto de dosis subletales de abamectina y sus costos de resistencia en la biología y desarrollo de Plutella xylostella.

\section{Materiales y métodos}

Insectos. La línea de campo se recolectó en parcelas comerciales de brócoli ubicadas en el municipio de Abasolo $\left(20^{\circ} 27^{\prime} 10.5^{\prime \prime} \mathrm{N} 101^{\circ} 35^{\prime} 14.0^{\prime \prime} \mathrm{E}\right)$ en el Estado de Guanajuato, México y fue criada en condiciones de laboratorio en jaulas entomológicas de $60 \mathrm{~cm}$ x $40 \mathrm{~cm}$ x $1 \mathrm{~cm}$. Para aumentar el nivel de resistencia se mantuvieron bajo presión de selección con el insecticida abamectina durante diez generaciones $\left(\mathrm{G}_{10}\right)$. Las larvas fueron alimentadas con plantas de brócoli de 50 días de edad, las cuales eran cambiadas cada 2 días y la de los adultos en una solución azucarada al $15 \%$, esto para asegurar el apareamiento y la ovoposición. Todas las etapas de desarrollo de la PDD se mantuvieron en condiciones controladas de $27 \pm 1{ }^{\circ} \mathrm{C}, 80 \pm 10 \% \mathrm{HR}, 16: 8 \mathrm{~h} \mathrm{~L}: \mathrm{O}$. Como línea susceptible se utilizaron individuos proporcionados por Instituto Nacional de Investigación Forestal Agrícola y Pecuaria (INIFAP), criados sin presión de selección de insecticidas desde 1996.

Bioensayos. Para determinar las concentraciones subletales, $\mathrm{CL}_{50}$ (mata el $50 \%$ de los individuos expuestos al insecticida) y $\mathrm{CL}_{20}$ (mata el $20 \%$ de individuos expuestos al insecticida) de abamectina se utilizó el método de inmersión de hojas (IRAC 2019), utilizando un diseño experimental completamente al azar con seis concentraciones y un testigo absoluto de agua más adherente. Se cortaron discos de hojas de Brassica oleracea var. Itálica Plenck de $6 \mathrm{~cm}$ de diámetro y se sumergieron durante $10 \mathrm{~s}$ en las diferentes concentraciones a evaluar. Las hojas se dejaron secar durante $1 \mathrm{~h}$ y luego se colocaron de forma individual en cajas Petri revestidas con papel absorbente humedecido con agua. Se transfirieron un total de 10 larvas de tercer instar (L3) por cada disco de hoja y se realizaron cuatro repeticiones por concentración. La mortalidad se evaluó 24 h después del inicio del experimento; las larvas que no reaccionaron cuando fueron estimuladas con un pincel en la parte dorsal se registraron como muertas. Una vez determinada la $\mathrm{CL}_{20}$ se aplicó durante las próximas 10 generaciones alimentando las larvas con plantas de $B$. oleracea tratadas con la $\mathrm{CL}_{20}$ de la generación anterior, hasta que no se presentaron en la respuesta al insecticida.

Efectos subletales y costos de la resistencia. Diez larvas de tercer instar de la línea resistente (LR) y de la línea susceptible (LS) se colocaron en disco de hoja tratado con la $\mathrm{CL}_{20}$ de cada línea en estudio, se dejaron alimentar por $24 \mathrm{~h} \mathrm{y}$, posteriormente, se cambió la hoja por otra no tratada y se observaron hasta alcanzar su fase de pupa y la emergencia de adultos y se registró el número de pupas formadas y adultos emergidos. El porcentaje de pupas formadas se estimó dividiendo el total de pupas obtenidas entre el número inicial de larvas y para el porcentaje de adultos emergidos se dividió el total de adultos entre el número inicial de pupas. Se utilizó un diseño experimental completamente al azar y 10 repeticiones por cada tratamiento; cada hoja con 10 larvas de tercer instar se consideró como una unidad experimental. Los adultos fueron se sexados y se conformaron 10 parejas, las cuales se colocaron en cajas Petri provistas de papel absorbente humedecido con agua y una hoja de $B$. oleracea var. itálica, se alimentaron con agua azucarada al $15 \%$ y se dejaron copular y ovipositar hasta que la última hembra murió. Diariamente se tomó el registro de los huevos ovipositados por hembra. Se tomaron 100 huevos al azar de las parejas formadas y se colocaron sobre discos de hojas de brócoli en cajas Petri revestidas con papel absorbente 
humedecido con agua, utilizando un diseño experimental completamente al azar con 10 repeticiones; cada unidad experimental consistió en un disco de hoja de brócoli con 10 huevos. Se registró la duración del desarrollo en cada una de las etapas larvales, así como el porcentaje de pupas formadas y adultos emergidos. En la etapa de pupa, se seleccionaron 10 pupas al azar de dos días de edad y se pesaron; en los adultos emergidos se evaluaron los efectos subletales, como proporción sexual, longevidad del adulto (hembra y macho) y fecundidad. La proporción sexual se calculó dividiendo el número de hembras entre el número de machos, obteniendo así hembras por macho; por su parte la longevidad de los adultos se estimó mediante los días de vida del adulto desde que emergía de la pupa hasta su muerte. Durante la fase experimental los insectos se mantuvieron en condiciones ambientales controladas de $27 \pm 1{ }^{\circ} \mathrm{C}, 80 \pm 10 \% \mathrm{HR}, 16: 8 \mathrm{~h} \mathrm{~L}: \mathrm{O}$.

Análisis de resultados. Los datos de los bioensayos fueron corregidos con la fórmula de Abbott (1925) y los resultados se sometieron a un análisis probit (Finney 1971) para obtener la curva de respuesta concentración-mortalidad, utilizando el programa SAS System for Windows ver 9.0 (SAS Institute Inc 2004). Con los valores de las variables de desarrollo se realizó un análisis de varianza (ANOVA) y, posteriormente, se aplicó la prueba de Tukey $(\alpha<0,05)$, para la separación de las medias. Los análisis estadísticos fueron realizados con el programa R-studio, versión 3.3.1 (R CORE TEAM 2020).

\section{Resultados}

La presión de selección de abamectina sobre una línea de campo de $P$. xylostella estimuló un desarrolló en la resistencia de 33,39 veces más en comparación a la LS (Tabla 1). Se reporta una $\mathrm{CL}_{50}$ de $1,31 \mathrm{ppm}$ para la LS y de $1,33 \mathrm{ppm}$ para la primera generación $\left(\mathrm{G}_{1}\right)$ de la línea de campo, después de realizar aplicaciones de una $\mathrm{CL}_{20}$ de abamectina durante diez generaciones $\left(\mathrm{G}_{10}\right)$ en la línea de campo la $\mathrm{CL}_{50}$ aumentó a $43,75 \mathrm{ppm}$.
Tabla 1. Concentración letal media $\left(\mathrm{CL}_{50}\right)$ de abamectina en diferentes generaciones; $\mathrm{G}_{1}$ primera generación y $\mathrm{G}_{10}$ decima generación de Plutella xylostella.

\begin{tabular}{cccccc}
\hline Generación & $\mathbf{C L}_{\mathbf{5 0}}$ & LFS-LFI & $\mathbf{C L}_{\mathbf{2 0}}$ & Ec. Predicción & P.R $^{\mathbf{1}}$ \\
\hline $\mathrm{LS}$ & 1,310 & $0,70-2,21$ & 0,21 & $\mathrm{y}=-0,12+1,06$ & - \\
$\mathrm{G}_{1}$ & 1,330 & $0,58-2,76$ & 0,28 & $\mathrm{y}=-0,15+1,25$ & 1,01 \\
$\mathrm{G}_{10}$ & 43,757 & $41,36-46,22$ & 28,43 & $\mathrm{y}=-7,37+4,49$ & 33,39 \\
\hline
\end{tabular}

${ }^{1}$ Proporción de resistencia; LFS = Limite fiducial superior; LFI = Limite fiducial inferior; $\mathrm{LS}=$ Línea susceptible.

Los efectos subletales y costos de la resistencia a la abamectina muestran diferencias significativas en los tratamientos evaluados para la generación progenitora en el porcentaje de pupas $\left(\mathrm{F}=24,16 ; \mathrm{P}=9,66 \mathrm{e}^{-09}\right)$ y peso de pupas $(\mathrm{F}=93,04$; $\left.\mathrm{P}=2 \mathrm{e}^{-16}\right)$. Asi mismo, en los descendientes se muestran diferencias significativas en el porcentaje de pupas $(\mathrm{F}=16,41$; $\left.\mathrm{P}=6,94 \mathrm{e}^{-07}\right)$ y peso de pupas $\left(\mathrm{F}=38,45 ; \mathrm{P}=2,55 \mathrm{e}^{-11}\right)$ de $P$. xylostella (Figura 1). En el porcentaje de pupas formadas la LS presentó el valor más alto con más de un $95 \%$ y la línea resistente con aplicación $\left(\mathrm{LRCL}_{20}\right)$ reportó el menor valor por debajo del $50 \%$ para el caso de la generación progenitora. Sin embargo, el efecto de la resistencia y la aplicación de abamectina se reflejó en los descendientes al reportar valores mayores del $90 \%$ para la línea resistente (LR), $\mathrm{LRCL}_{20}$ y LS. Por otra parte, la aplicación de abamectina en la LS afectó a los descendientes al disminuir el porcentaje de pupas al 50 $\%$. Para la generación de progenitores la $\mathrm{LRCL}_{20}$ incrementó más de tres veces el peso de las pupas en comparación a la LR y LS. Sin embargo, la aplicación sobre la LS, provocó una disminución del $50 \%$ en el peso de pupas en comparación a la LS. Efectos similares se reportan sobre los descendientes al aumentar el peso de pupas más del doble en la $\mathrm{LRCL}_{20} \mathrm{y}$ disminución en más del $50 \%$ en $\mathrm{LSCL}_{20}$ en comparación a las líneas sin tratar.
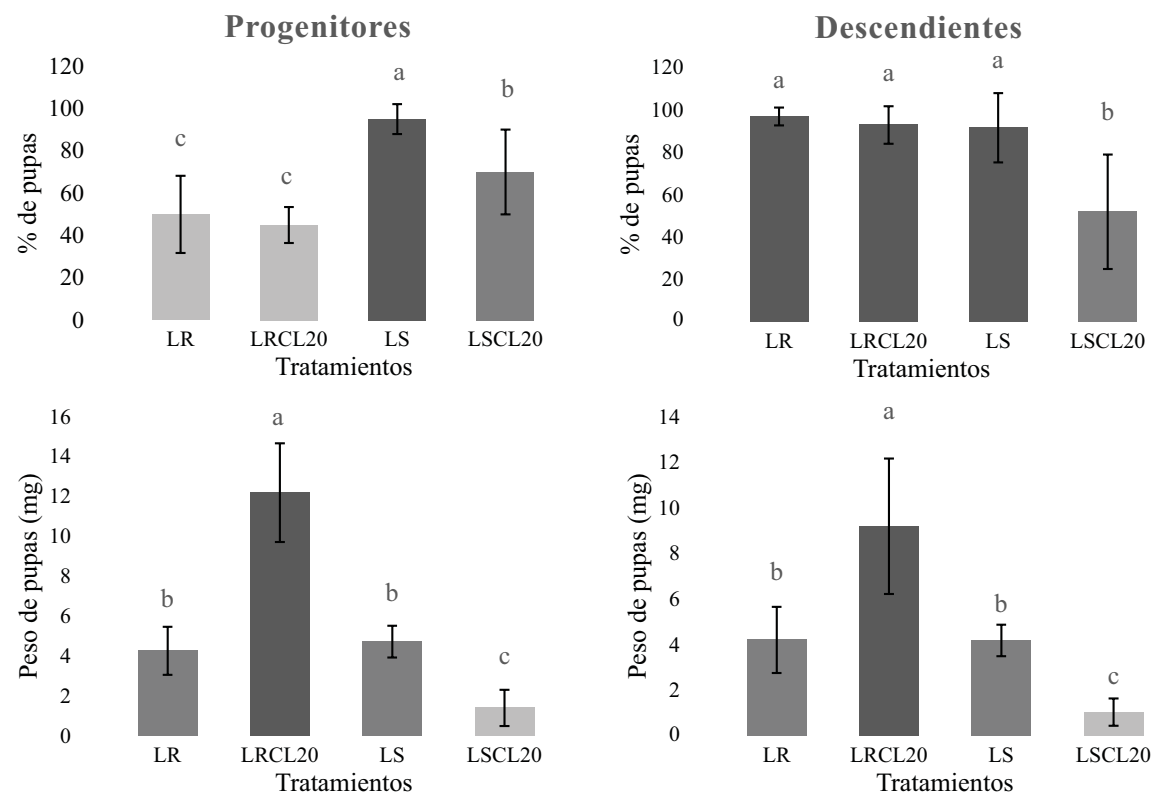

Figura 1. Efectos subletales de la abamectina en el porcentaje pupal y peso de pupas de Plutella xylostella. Medias seguidas de la misma letra no presentan diferencia significativa (Tukey, $\alpha<0,05$ ) $\mathrm{LS}=$ Línea resistente; $\mathrm{LS}=$ Línea susceptible $; \mathrm{LRCL}_{20}=$ Línea resistente con aplicación; $\mathrm{LSCL}_{20}=$ Línea susceptible con aplicación. 
En el porcentaje de adultos emergidos en la generación de progenitores no se presentaron diferencias estadísticamente significativas; sin embargo, en los descendientes sí se presenta un efecto de la resistencia $(\mathrm{F}=5,68 ; \mathrm{P}=0,002)$ y la aplicación de $\mathrm{CL}_{20}$ al incrementar el porcentaje de adultos emergidos con 98 y $96,78 \%$ para LR y $\mathrm{LRCL}_{20}$, respectivamente. En lo que se refiere a la proporción sexual, en ninguna de las dos generaciones se presentaron diferencias significativas entre las líneas en estudio, considerando que la resistencia y la aplicación de $\mathrm{CL}_{20}$ de abamectina no tiene un efecto importante en la proporción de sexos en P. xylostella (Tabla 2).

La biología de $P$. xyllostela fue afectada por la resistencia y la aplicación de $\mathrm{CL}_{20}$ de abamectina, la cual se midió mediante la oviposición de huevos por hembra por día (HHD) (Tabla 3). Las hembras progenitoras de la LR ovipositaron mas huevos a lo largo de su vida con 220,20 huevos y un promedio de 32,49 HHD; sin embargo, al aplicar $\mathrm{CL}_{20}$ de abamectina en individuos resistentes $\left(\mathrm{LRCL}_{20}\right)$, esto propició una reducción en la ovoposición con un total de 177,40 huevos y una media de 31,01 HHD, por su parte la LS reporta 34,04 HHD y un total de 140 huevos. Las hembras descendientes también fueron afectas por la resistencia y la aplicación de $\mathrm{CL}_{20}$ de abamectina, pero en este caso de manera negativa al disminuir su total de huevos en comparación con las hembras progenitoras con $172,60,116,20$ y 90,20 para $\mathrm{LSCL}_{20}$, $\mathrm{LRCL}_{20}$ y LR, respectivamente.

El tiempo de desarrollo de cada una de las fases de $P$. xylostella fueron afectados de manera importante a causa de la resistencia y la exposición a $\mathrm{CL}_{20}$ de abamectina (Tabla 4). Se presentaron diferencias significativas entre las líneas en estudio en cada una de las fases de desarrollo. Se observó un incremento en el tiempo de desarrollo de cada una de las fases en la LR y en $\mathrm{LRCL}_{20}$ en comparación a la LS, siendo las fases de larva 2, larva 3 y larva 4 las de mayor incremento de tiempo de desarrollo, por su parte el tiempo de desarrollo de los machos aumentó en más de 2,5 días y el de las hembras más de 1,5 días, asegurando así una mayor copulación y oviposición de los adultos. El tiempo total de desarrollo desde huevo hasta la muerte del adulto aumentó significativamente en 5,88 y 7,29 días para la $\mathrm{LR}$ y $\mathrm{LRCL}_{20}$, respectivamente, en comparación a la línea susceptible.

\section{Discusión}

Estudios previos mencionan que $P$. xylostella presenta una gran habilidad para desarrollar resistencia a insecticidas elaborados a base de microrganismos como spinosad, benzoato de enamectina y abamectina (Meghana et al. 2018). En esta

Tabla 2. Efectos subletales de abamectina en el porcentaje de adultos emergidos y proporción sexual de Plutella xylostella.

\begin{tabular}{lcccc}
\hline \multirow{2}{*}{ Trat $^{1}$} & \multicolumn{2}{c}{ Progenitores } & \multicolumn{2}{c}{ Descendientes } \\
\cline { 2 - 5 } & \% de Adultos & $\begin{array}{c}\text { Proporción } \\
\text { sexual }\end{array}$ & \% de Adultos & $\begin{array}{c}\text { Proporción } \\
\text { sexual }\end{array}$ \\
\hline $\mathrm{LS}$ & $81,00 \pm 11,97 \mathrm{a}$ & $1,36 \pm 0,92 \mathrm{a}$ & $85,57 \pm 7,53 \mathrm{ab}$ & $1,92 \pm 1,25 \mathrm{a}$ \\
$\mathrm{LSCL}_{20}$ & $82,44 \pm 17,23 \mathrm{a}$ & $1,61 \pm 0,56 \mathrm{a}$ & $75,19 \pm 26,65 \mathrm{~b}$ & $1,27 \pm 1,77 \mathrm{a}$ \\
$\mathrm{LR}^{1}$ & $73,15 \pm 19,72 \mathrm{a}$ & $1,07 \pm 0,83 \mathrm{a}$ & $98,00 \pm 3,49 \mathrm{a}$ & $1,25 \pm 0,35 \mathrm{a}$ \\
$\mathrm{LRCL}_{20}$ & $69,62 \pm 1,12 \mathrm{a}$ & $1,37 \pm 0,45 \mathrm{a}$ & $96,78 \pm 5,21 \mathrm{a}$ & $1,09 \pm 0,69 \mathrm{a}$ \\
\hline
\end{tabular}

Medias seguidas de la misma letra no presentan diferencia significativa (Tukey, $\alpha<0,05$ ); ${ }^{1}$ Hembras por macho. LR = Línea resistente; LS = Línea susceptible; $\mathrm{LRCL}_{20}=$ Línea resistente con aplicación; $\mathrm{LSCL}_{20}=$ Línea susceptible con aplicación.
Tabla 3. Efectos subletales de abamectina en la oviposición de hembras de Plutella xylostella.

\begin{tabular}{lcccc}
\hline \multirow{2}{*}{ Trat $^{1}$} & \multicolumn{2}{c}{ Progenitores } & \multicolumn{2}{c}{ Descendientes } \\
\cline { 2 - 5 } & HHD $^{2}$ & Total & HHD $^{2}$ & Total \\
\hline LS & $34,04 \pm 1,55$ a & $140,20 \pm 8,10$ c & $27,16 \pm 3,55$ b & $145,80 \pm 15,46$ a \\
LSCL $_{20}$ & $25,84 \pm 3,18$ b & $204,80 \pm 8,34$ ab & $42,53 \pm 3,53$ a & $172,60 \pm 23,04$ a \\
LR & $32,49 \pm 0,89$ a & $220,20 \pm 12,47$ a & $19,36 \pm 2,70$ c & $116,20 \pm 12,41$ b \\
LRCL $_{20}$ & $31,01 \pm 1,31$ a & $177,40 \pm 41,20$ bc & $29,45 \pm 3,53$ b & $90,20 \pm 4,81$ b \\
\hline
\end{tabular}

Medias seguidas de la misma letra no presentan diferencia significativa (Tukey, $\alpha<0,05)$; Progenitores: HHD $\left(\mathrm{F}=16,73 ; \mathrm{P}=3,45 \mathrm{e}^{-05}\right)$; Total $(\mathrm{F}$ $=12,48 ; \mathrm{P}=0,0001)$, Descendientes: HHD $\left(\mathrm{F}=46,48 ; \mathrm{P}=4,01 \mathrm{e}^{-08}\right)$; Total $\left(\mathrm{F}=26,96 ; \mathrm{P}=1,71 \mathrm{e}^{-06}\right) ; \mathrm{HHD}=$ Huevos por hembra por día; $\mathrm{LS}=$ Línea resistente; $\mathrm{LS}=$ Línea susceptible; $\mathrm{LRCL}_{20}=$ Línea resistente con aplicación; $\mathrm{LSCL}_{20}=$ Línea susceptible con aplicación.

investigación se confirma dicha capacidad al obtener un factor de resistencia de 33,39 veces al realizar una presión de selección de abamectina en diez generaciones. Zhou et al. (2010) reportan un incremento en la susceptibilidad de 5,80 veces para este mismo insecticida bajo una selección de resistencia en 11 generaciones de P. xylostella. Por su parte Wang y Wu (2014) obtuvieron un factor de resistencia de 11,50 veces para una línea recolectada en campo y mantenida bajo presión de selección durante 21 generaciones.

La resistencia a abamectina y la aplicación de dosis subletales presenta alteraciones en el desarrollo y biología de P. xyllostella, al aumentar el porcentaje de pupas formadas de una generación a otra así como el peso de las mismas; Wang y Wu (2014) reportan una disminución en formación de pupas y un peso de pupas en una línea resistente a este insecticida; resultados similares se documentaron al aplicar dosis subletales de spinosad (Yin et al. 2008) y clorantraniliprol (Han et al. 2012) que reducen significativamente la tasa de pupación y el peso de la pupas. Por su parte Moustafa et al. (2016) mencionan que la aplicación de dosis subletales del benzoato de emamectina (insecticida derivado de la abamectina)

Tabla 4. Efectos subletales de abamectina en el tiempo de desarrollo de Plutella xylostella.

\begin{tabular}{lcccc}
\hline \multirow{2}{*}{ Fase $^{1}$} & \multicolumn{4}{c}{ Tratamientos } \\
\cline { 2 - 5 } & $\mathbf{L S}$ & $\mathbf{L S C L}_{\mathbf{2 0}}$ & $\mathbf{L R}$ & $\mathbf{L R C L}_{\mathbf{2 0}}$ \\
\hline Huevo-L1 & $3,55 \pm 0,39 \mathrm{~b}$ & $2,87 \pm 0,30 \mathrm{c}$ & $4,23 \pm 0,50 \mathrm{a}$ & $3,73 \pm 0,74 \mathrm{ab}$ \\
L1-L2 & $1,08 \pm 0,17 \mathrm{~b}$ & $1,84 \pm 0,60 \mathrm{a}$ & $1,61 \pm 0,38 \mathrm{ab}$ & $1,47 \pm 0,69 \mathrm{ab}$ \\
L2-L3 & $1,83 \pm 0,33 \mathrm{~b}$ & $1,86 \pm 0,28 \mathrm{~b}$ & $3,13 \pm 0,60 \mathrm{a}$ & $3,49 \pm 0,69 \mathrm{a}$ \\
L3-L4 & $1,91 \pm 0,41 \mathrm{ab}$ & $1,59 \pm 0,34 \mathrm{~b}$ & $2,28 \pm 0,37 \mathrm{a}$ & $2,23 \pm 0,21 \mathrm{a}$ \\
L4-Pupa & $1,79 \pm 0,27 \mathrm{~b}$ & $1,50 \pm 0,52 \mathrm{~b}$ & $2,76 \pm 0,20 \mathrm{a}$ & $2,38 \pm 0,30 \mathrm{a}$ \\
Pupa-Adulto & $4,05 \pm 0,90 \mathrm{c}$ & $3,00 \pm 0,50 \mathrm{~d}$ & $4,78 \pm 0,26 \mathrm{~b}$ & $5,83 \pm 0,29 \mathrm{~b}$ \\
Adulto & $3,57 \pm 0,47 \mathrm{c}$ & $5,73 \pm 0,75 \mathrm{a}$ & $4,90 \pm 0,62 \mathrm{~b}$ & $5,95 \pm 0,59 \mathrm{a}$ \\
Macho & $2,89 \pm 0,89 \mathrm{~b}$ & $6,26 \pm 2,12 \mathrm{a}$ & $5,10 \pm 0,99 \mathrm{a}$ & $5,80 \pm 0,78 \mathrm{a}$ \\
Hembra & $4,41 \pm 0,79 \mathrm{~b}$ & $5,20 \pm 1,03 \mathrm{ab}$ & $4,60 \pm 0,51 \mathrm{~b}$ & $5,90 \pm 0,88 \mathrm{a}$ \\
Total & $17,81 \pm 0,99 \mathrm{c}$ & $18,41 \pm 1,45 \mathrm{c}$ & $23,69 \pm 1,21 \mathrm{~b}$ & $25,10 \pm 0,85 \mathrm{a}$ \\
\hline
\end{tabular}

Medias seguidas de la misma letra por cada fila no presentan diferencia significativa (Tukey, $\alpha<0,05)$; Huevo-L1 $\left(\mathrm{F}=11,85 ; \mathrm{P}=1,51 \mathrm{e}^{-05}\right)$; Tiempo de desarrollo de un estadio larval a otro: L1-L2 $(\mathrm{F}=3,99 ; \mathrm{P}=0,01)$; L2-L3 ( $\mathrm{F}$ $\left.=28,23 ; \mathrm{P}=1,45 \mathrm{e}^{-09}\right), \mathrm{L} 3-\mathrm{L} 4(\mathrm{~F}=8,37 ; \mathrm{P}=0,0002) ; \mathrm{L} 4-\mathrm{L} 5(\mathrm{~F}=26,53 ; \mathrm{P}$ $\left.=3,12 \mathrm{e}^{-09}\right)$; Pupa-Adulto $\left(\mathrm{F}=58,05 ; \mathrm{P}=7,22 \mathrm{e}^{-14}\right)$; Adulto $(\mathrm{F}=29,98 ; \mathrm{P}=$ $\left.6,76 \mathrm{e}^{-10}\right)$; Hembras $(\mathrm{F}=6,14 ; \mathrm{P}=0,001)$; Macho $\left(\mathrm{F}=12,86 ; \mathrm{P}=7,28 \mathrm{e}^{-06}\right)$; Total $\left(\mathrm{F}=101,9 ; \mathrm{P}=2 \mathrm{e}^{-16}\right) ;{ }^{1}$ valores en días; $\mathrm{LS}=$ Línea resistente; $\mathrm{LS}=$ Línea susceptible; $\mathrm{LRCL}_{20}=$ Línea resistente con aplicación; $\mathrm{LSCL}_{20}=$ Línea susceptible con aplicación. 
disminuye el porcentaje de pupas y aumenta el peso de las mismas para Mamestra brassicae L., 1758 (Lepidoptera: Noctuidae).

La emergencia de adultos de Plutella xylostella en la primera generación fue afectada por la resistencia y la exposición de abamectina al disminuir su porcentaje en comparación a la LS, sim embargo para la siguiente generación aumentó de manera considerable la tasa de adultos emergidos, estos resultados difieren a los reportados por Zibaee y Esmaeily (2017) quienes evaluaron los efectos subletales de abamectina sobre Tuta absoluta que al ser expuesta a dosis subletales la emergencia de adultos no presentó cambios de una generación a otra. Por su parte Moustafa et al. (2016) muestran una reducción el porcentaje de emergencia al exponer larvas de tercer instar de $P$. xylostella a los insecticidas spinosad y benzoato de enamectina. La restauración de una plaga de insectos se puede dar de manera gradual de generación en generación; sin embargo, el punto final de un efecto subletal va depender de la especie de insecto, el tipo de aplicación, dosis y la clase de insecticida (Zibaee y Esmaeily 2017).

La combinación de la resistencia presente en $P$. xylostella y la aplicación de dosis subletales juegan un factor importante en la formación y peso de pupas, lo que podría generar mayor número de adultos resistentes y con un mayor apetito reproductivo.

Estudios previos han demostrado que la reducción en la fecundidad que ocurre en un insecto se relaciona a los cambios fisiológicos y morfológicos en ambos sexos (machos y hembras) (Sáenz-de-Cabezón et al. 2006). La biosíntesis de feromonas sexuales en los insectos implica una coordinación compleja de actividades fisiológicas que están bajo controles hormonales y neuronales (Rafaeli 2011; Bloch et al. 2013). Las hembras de $P$. xylostella producen menos feromona sexual y llegan a mostrar un nivel más bajo de comportamiento de llamada en cambio los machos resistentes muestran una mayor capacidad de respuesta a la mezcla de feromonas sexuales de hembras (Xu et al. 2010).

El ciclo de vida de $P$. xylostella fue afectado a causa de la resistencia y a la exposición de dosis subletal, al incrementar los días de vida en comparación a la LS. Se conoce que las concentraciones subletales de indoxacarb (Wang et al. 2011) y clorantraniliprol (Guo et al. 2013) inhiben el desarrollo de $P$. xylostella. Zibaee y Esmaeily (2017) mencionan que la aplicación de abamectina en Tuta absoluta no afecta el tiempo de desarrollo de huevo hasta la muerte del adulto. La aplicación de dosis subletales de abamectina y sus efectos sobre su desarrollo y biología reportados en este estudio se pueden asociar en gran medida al modo de acción de este insecticida, que se encuentran relaciona con el receptor GABA, que interfiere con la coordinación motora de los músculos involucrados en la alimentación y el movimiento de los insectos (Deecher et al. 1990; Zibaee y Esmaeily 2017).

La respuesta de los insectos a los insecticidas (dosis-respuesta) permite determinar la aparición de hormesis (Qu et al. 2015), fenómeno causado por un factor estresante (plaguicida), provocando una respuesta diferente entre y dosis bajas y altas (Jager et al. 2013). Las dosis que causan respuestas horméticas tienen un rango limitado y, por lo general, están por debajo de la concentración letal, aunque no siempre es el caso en la respuesta de los insectos a los plaguicidas (Cutler 2013). La hormesis inducida por plaguicidas es el principal problema para el manejo de la resistencia a plaguicidas debido a la exposición constante de insectos resistentes a dosis subletales de plaguicidas en el campo (Guedes et al. 2010). Esto representa un problema para el manejo de poblaciones resistentes porque las dosis de campo pueden no controlar a los insectos-plaga y aumentar el crecimiento de la población, además de aumentar el desarrollo de resistencia de las plagas (Guedes y Clutler 2013). Sin embargo, la hormesis no solo se limita al estrés por plaguicidas (Calabrese y Blain 2005; Cluter 2013), existen otros factores de estrés como la temperatura, la dieta y la radiación (Mattson y Calabrese 2010; López-Martínez y Hahn 2012). Las combinaciones de estos factores pueden tener costos importantes en la aptitud biológica, el crecimiento y la resistencia a los plaguicidas (Piiroinen et al. 2013).

La aplicación de dosis subletales de abamectina afecta la biología y desarrollo de $P$. xylostella lo que repercutiría en una mayor número de individuos por ciclo, mayor tiempo de desarrollo y mayor tasa de ovoposición dando paso a posibles brotes de la plaga lo que aumentaría el número de aplicaciones por ciclo y aumentado los costos de control, por lo que se recomienda disminuir las aplicaciones de abamectina o insecticidas de un modo de acción similar y realizar rotación con diferente grupo toxicológico para evitar el desarrollo de resistencia

\section{Conclusiones}

Plutella xylostella presenta una gran capacidad de desarrollar resistencia a abamectina en pocas generaciones al aumentar su proporción de resistencia en más de 30 veces en comparación a la línea susceptible.

La resistencia y la aplicación de concentraciones subletales de abamectina $\left(\mathrm{CL}_{20}\right)$ sobre Plutella xylostella afectan de manera importante su biología y desarrollo, al aumentar el número de adultos por generación, peso de pupas, número de huevos por hembra y al alargar su ciclo de vida en más siete días, generando un mayor número de individuos por generación y ciclo de vida más largo, además de estar estimulando el desarrollo de resistencia.

\section{Literatura citada}

ABBOTT, W. S. 1925. A method of computing the effectiveness of an insecticide. Journal of Economic Entomology 18 (2): 265267. https://doi.org/10.1093/jee/18.2.265a

ARTHROPOD PESTICIDE RESISTANCE DATABASE. 2020. Plutella xylostella. APRD. Disponible en: https://www. pesticideresistance.org/display.php?page $=$ species \&arId $=571$ [fecha revisión: 20 abril 2020].

BANKS, J. E.; VARGAS, R. I.; ACKLEH, A. S.; STARK, J. D. 2017. Sublethal effects in pest management: a surrogate species perspective on fruit fly control. Insects 8 (3): 1-6. https://doi. org/10.3390/insects 8030078

BLOCH, G.; HAZAN, E.; RAFAELI, A. 2013. Circadian rhythms and endocrine functions in adult insects. Journal of Insect Physiology 59 (1): 56-69. https://doi.org/10.1016/j.jinsphys.2012.10.012

BOWMAN, D. D. 2021. Georgis' parasitology for veterinarians. 11 th ed. Elsilver inc. St. Louis, Missouri. EE.UU. 287 p. https:// books.google.com.mx/books?hl=es\&lr=\&id=2576DwAAQBA J\&oi=fnd\&pg=PP1\&ots=2aVHC9TK-_\&sig=KqUq4GieK-i8pPcgroNtNeDnmY\&redir_esc $=\mathrm{y} \# \mathrm{v}=$ onepage $\& \mathrm{q} \& \mathrm{f}=$ false

CALABRESE, E. J.; BALDWIN, L. A. 2001. Hormesis: a generalizable and unifying hypothesis. Critical Reviews in Toxicology 31 (4-5): 353-424. https://doi.org/10.1080/20014091111730

CALABRESE, E. J.; BLAIN, R. 2005. The occurrence of hormetic dose responses in the toxicological literature, the hormesis 
database: an overview. Toxicology and applied pharmacology 202 (3): 289-301. https://doi.org/10.1016/j.taap.2004.06.023

CORDEIRO, E. M. G.; MOURA, I. L. T.; FADINI, M. A. M.; GUEDES, R. N. C. 2013. Beyond selectivity: are behavioral avoidance and hormesis likely causes of pyrethroid-induced outbreaks of the southern red mite Oligonychus ilicis? Chemosphere 93 (6): 1111-1116. https://doi.org/10.1016/j. chemosphere.2013.06.030

CUTLER, G. C. 2013. Insects, insecticides and hormesis: evidence and considerations for study. International Dose-Response Society $11 \quad$ (2): 154-177. https://doi.org/10.2203/doseresponse.12-008.Cutler

DE CASTRO, A. A.; CORRÊA, A. S.; LEGASPI, J. C.; GUEDES, R. N. C.; SERRÃ̃O, J. E.; ZANUNCIO, J. C. 2013. Survival and behavior of insecticide-exposed predators Podisus nigrispinus and Supputius cincticeps (Heteroptera: Pentatomidae). Chemosphere 93 (6): 1043-1050. https://doi.org/10.1016/j. chemosphere.2013.05.075

DEECHER, D. C.; BREZNER, J.; TANENBAUM, S. W. 1990. Sublethal effects of avermectin and milbemycin on the gypsy moth (Lepidoptera: Lymantriidae). Journal of Economic Entomology 83 (3): 710-714. https://doi.org/10.1093/jee/83.3.710

FINNEY, D. J. 1971. Probit analysis. 3rd Edition Cambridge, University Press. New York, EE. UU. 333 p. https://doi.org/10.1002/ jps.2600600940

FURLONG, M. J.; WRIGHT, D. J.; DOSDALL, L. M. 2013. Diamondback moth ecology and management: problems, progress, and prospects. Annual Review of Entomology 58 (1): 517-541. https://doi.org/10.1146/annurev-ento-120811-153605

GARCÍA SALAZAR, B.; HERNÁNDEZ MORENO, D.; SOLER RODRÍGUEZ, F.; PÉREZ-LÓPEZ, M. 2011. Empleo de ivermectina como parasiticida en ovino: posibles efectos tóxicos y repercusiones ambientale. Anales de veterinaria de Murcia 27: 23-32. https://doi.org/10.6018/j/160111

GRZYWACZ, D.; ROSSBACH, A.; RAUF, A., RUSSELL, D. A.; SRINIVASAN, R.; SHELTON, A. M. 2010. Current control methods for diamondback moth and other brassica insect pests and the prospects for improved management with lepidopteran-resistant $\mathrm{Bt}$ vegetable brassicas in Asia and Africa. Crop Protection 29 (1): 68-79. https://doi.org/10.1016/j. cropro.2009.08.009

GUEDES, R. N. C.; CUTLER, G. C. 2013. Insecticide- induced hormesis and arthropod pest management. Pest Management Science 70 (5): 690-697. https://doi.org/10.1002/ps.3669

GUEDES, N. M. P.; TOLLEDO, J.; CORRÊA, A. S.; GUEDES, R. N. C. 2010. Insecticide-induced hormesis in an insecticide-resistant strain of the maize weevil, Sitophilus zeamais. Journal of Applied Entomology 134 (2): 142-148. https://doi.org/10.1111/ j.1439-0418.2009.01462.x

GUO, L.; DESNEUX, N.; SONODA, S.; LIANG, P.; HAN, P.; GAO, X-W. 2013. Sublethal and transgenerational effects of chlorantraniliprole on biological traits of the diamondback moth, Plutella xylostella L. Crop Protection 48: 29-34. https:// doi.org/10.1016/j.cropro.2013.02.009

HAN, W.; ZHANG, S.; SHEN, F.; LIU, M.; REN, C.; GAO, X. 2012. Residual toxicity and sublethal effects of chlorantraniliprole on Plutella xylostella (Lepidoptera: Plutellidae). Pest Management Science 68 (8): 1184-1190. https://doi.org/10.1002/ps.3282

IRAC (INSECTICIDE RESISTANCE ACTION COMMITTEE). 2019. Disponible en línea: https://irac-online.org/methods/plutella-xylostella-larvae/ (última consulta 8 de marzo 2019).

JAGER, T.; BARSI, A.; DUCROT, V. 2013. Hormesis on life-history traits: is there such thing as a free lunch? Ecotoxicology 22: 263-270. https://doi.org/10.1007/s10646-012-1022-0

LÓPEZ-MARTINEZ, G.; HAHN, D. A. 2012. Short-term anoxic conditioning hormesis boosts antioxidant defenses, lowers oxidative damage following irradiation and enhances male sexual performance in the Caribbean fruit fly, Anastrepha suspensa.
Journal of Experimental Biology 215 (12): 2150-2161. https:// doi.org/10.1242/jeb.065631

MATTSON, M. P.; CALABRESE, E. J. 2010. Hormesis: what it is and why it matters. pp. 1-13. En: Mattson, M. P.; Calabrese, E. J. (Eds.). Hormesis: A Revolution in Biology, Toxicology and Medicine. 1st Edition. Springer. New York, EE. UU. 213 p. https://doi.org/10.1007/978-1-60761-495-1

MEGHANA, C.; JAYAPPA, J.; REDDY, N. A.; DEVAPPA, V.; SRIDHAR, V.; KATTEGOUDAR, J. 2018. Assessing susceptibility of diamondback moth, Plutella xylostella (Lepidoptera: Plutellidae) population of different geographic region to selected newer insecticides. Journal of Entomology and Zoology Studies 6 (1): 320-327. http://www.entomoljournal.com/archives/ ?year $=2018 \&$ vol $=6 \&$ issue $=1 \&$ part $=$ E\&ArticleId $=2972$

MEMARIZADEH, N.; GHADAMYARI, M.; ZAMANI, P.; SAJEDI, R. H. 2013. Resistance mechanisms to abamectin in Iranian populations of the two-spotted spider mite, Tetranychus urticae Koch (Acari: Tetranychidae). Acarologia 53 (3): 235-246. https://doi.org/10.1051/acarologia/20132093

MOUSTAFA, M. A. M.; KÁKAI, Á.; AWAD, M.; FÓNAGY, A. 2016. Sublethal effects of spinosad and emamectin benzoate on larval development and reproductive activities of the cabbage moth, Mamestra brassicae L. (Lepidoptera: Noctuidae). Crop Protection 90: 197-204. https://doi.org/10.1016/j. cropro.2016.09.004

PIIROINEN, S.; LYYTINEN, A.; LINDSTRÖM, L. 2013. Stress for invasion success? Temperature stress of preceding generations modifies the response to insecticide stress in an invasive pest insect. Evolutionary Applications 6 (2): 313-323. https://doi. org/10.1111/eva.12001

QU, Y.; XIAO, D.; LI, J.; CHEN, Z.; BIONDI, A.; DESNEUX, N.; GAO, X.; SONG, D. 2015. Sublethal and hormesis effects of imidacloprid on the soybean aphid Aphis glycines. Ecotoxicology 24 (3): 479-487. https://doi.org/10.1007/s10646-014-1396-2

R CORE TEAM. 2020. R: A language and environment for statistical computing. Foundation for Statistical Computing, Vienna, Austria. https://www.R-project.org/

RAFAELI, A. 2011. Moth sex-pheromone production: Biosynthetic pathways, regulatory physiology, inhibitory processes and disruption. pp 115-143. In: Cauterruccio, L. (Ed.). Moths: types, ecological significance and control. 1st Edition. Nova Science Publisher, Inc. New York, EE. UU. 286 p.

SABER, M.; AHMADI, Z.; MAHDAVINIA, G. 2018. Sublethal effects of spirodiclofen, abamectin and pyridaben on life-history traits and life-table parameters of two-spotted spider mite, Tetranychus urticae (Acari: Tetranychidae). Experimental and Applied Acarology 75 (1): 55-67. https://doi.org/10.1007/ s10493-018-0226-2

SÁENZ-DE-CABEZÓN, F. J.; PÉREZ-MORENO, I.; ZALOM, F. G.; MARCO, V. 2006. Effects of lufenuron on Lobesia botrana (Lepidoptera: Tortricidae) egg, larval, and adult stages. Journal of Economic Entomology 99 (2): 427-431. https://doi. org/10.1093/jee/99.2.427

SARAN, R. K.; ZIEGLER, M.; KUDLIE, S.; HARRISON, D.; LEVA, D. M.; SCHERER, C.; COFFELT, M. A. 2014. Behavioral effects and tunneling responses of eastern subterranean termites (Isoptera: Rhinotermitidae) exposed to chlorantraniliprole-treated soils. Journal of Economic Entomology 107(5): 1878-1889. https://doi.org/10.1603/EC11393

SAS Institute Inc. 2004. SAS/STAT ® 9.1 User's Guide. Cary, NC: SAS Institute Inc. 3703-3797 pp. https://support.sas.com/ documentation/onlinedoc/91pdf/sasdoc_91/stat_ug_7313.pdf 
WANG, G.; HUANG, X.; WEI, H.; FADAMIRO, H. Y. 2011. Sublethal effects of larval exposure to indoxacarb on reproductive activities of the diamondback moth, Plutella xylostella (L.) (Lepidoptera: Plutellidae). Pesticide Biochemistry and Physiology 101 (3): 227-231. https://doi.org/10.1016/j.pestbp.2011.09.010

WANG, R.; WU, Y. 2014. Dominant fitness costs of abamectin resistance in Plutella xylostella. Pest Management Science 70 (12): 1872-1876. https://doi.org/10.1002/ps.3741

WANG, X.; WU, Y. 2012. High levels of resistance to chlorantraniliprole evolved in field populations of Plutella xylostella. Journal of Economic Entomology 105 (3): 1019-1023. https://doi. org/10.1603/EC12059

XU, Z.; CAO, G-C.; DONG, S-L. 2010. Changes of sex pheromone communication systems associated with tebufenozide and abamectin resistance in diamondback moth, Plutella xylostella (L.). Journal of Chemical Ecology 36: 526-534. https://doi. org/10.1007/s10886-010-9785-3

YIN, X-H.; WU, Q-J.; LI, X-F.; ZHANG, Y-J.; XU, B-Y. 2008. Sublethal effects of spinosad on Plutella xylostella (Lepidoptera: Yponomeutidae). Crop Protection 27 (10): 1385-1391. https:// doi.org/10.1016/j.cropro.2008.05.008

ZHOU, X-M.; WU, Q-J.; ZHANG, Y-J.; BAI, L-Y.; HUANG, X-Y. 2010. Effects of abamectin selection on the genetic differentiation within Plutella xylostella (Lepidoptera: Plutellidae) based on amplified fragment length polymorphism. Insect Science 17 (4): 353-360. https://doi.org/10.1111/j.1744-7917.2009.01307.x

ZIBAEE, I.; ESMAEILY, M. 2017. Effect of sublethal doses of abamectin on demographic traits of tomato leafminer Tuta absoluta (Meyrick, 1917) (Lepidoptera: Gelechiidae). Journal of Plant Protection Research 57 (3): 256-267. https://doi. org/10.1515/jppr-2017-0036

\section{Origen y financiación}

Este manuscrito fue originado en el proyecto de investigación (No. 2116), titulado "Caracterización genética y enzimática de seis poblaciones de Plutella xylostella L. en relación a la resistencia a insecticidas en brócoli del Estado de Guanajuato". Este fue financiado por la Universidad Autónoma Agraria Antonio Narro, presupuesto asignado mediante convocatoria interna.

\section{Contribución de los autores}

José Francisco Rodríguez-Rodríguez: Recolección, cría y manejo del material biológico, realización de mediciones, interpretación de los análisis de datos y escritura del manuscrito.

Ernesto Cerna-Chávez: Análisis e interpretación de datos y escritura del manuscrito. Coordinación de las actividades de revisión del manuscrito.

Yisa María Ochoa-Fuentes: Análisis e interpretación de datos $y$ escritura del manuscrito.

Jerónimo Landeros-Flores: Ayuda en el análisis de los datos y escritura del manuscrito.

Luis Patricio Guevara-Acevedo: Manejo del material biológico, verificación taxonómica, colaboración en la interpretación de los análisis.

Hugo Cesar Cisneros-López: Manejo del material biológico y realización de mediciones. 